\title{
Differential MicroRNA-Signatures in Thyroid Cancer Subtypes
}

\author{
Krystal Santiago, Yan Chen Wongworawat $(\mathbb{D}$, and Salma Khan (iD) \\ Center for Health Disparities \& Molecular Medicine, Department of Pathology \& Human Anatomy, \\ Division of Biochemistry, Otolaryngology, Internal Medicine, Loma Linda University, School of Medicine, Loma Linda, CA, USA
}

Correspondence should be addressed to Salma Khan; salmakhan@llu.edu

Received 26 February 2020; Revised 5 May 2020; Accepted 18 May 2020; Published 3 June 2020

Academic Editor: Ozkan Kanat

Copyright (c) 2020 Krystal Santiago et al. This is an open access article distributed under the Creative Commons Attribution License, which permits unrestricted use, distribution, and reproduction in any medium, provided the original work is properly cited.

\begin{abstract}
Thyroid cancer is one of the most common endocrine cancers, with an increasing trend in the last few decades. Although papillary thyroid cancer is the most frequent subtype compared with follicular or anaplastic thyroid cancer, it can dedifferentiate to a more aggressive phenotype, and the recurrence rate is high. The cells of follicular adenomas and follicular carcinomas appear identical in cytology, making the preoperative diagnosis difficult. On the other hand, anaplastic thyroid cancer poses a significant clinical challenge due to its aggressive nature with no effective therapeutic options. In the past several years, the roles of genetic alterations of thyroid tumors have been documented, with a remarkable correlation between genotype and phenotype, indicating that distinct molecular changes are associated with a multistep tumorigenic process. Besides mRNA expression profiles, small noncoding microRNA (miRNA) expression also showed critical functions for cell differentiation, proliferation, angiogenesis, and resistance to apoptosis and finally activating invasion and metastasis in cancer. Several high-throughput sequencing studies demonstrate that miRNA expression signatures contribute clinically relevant information including types of thyroid cancer, tumor grade, and prognosis. This review summarizes recent findings on miRNA signatures in thyroid cancer subtypes.
\end{abstract}

\section{Introduction}

Thyroid cancer is the most common type of endocrine malignancy, and incidence has increased in the last few decades [1-4]. Although papillary thyroid cancer (PTC) is the most common differentiated thyroid cancer type, it is also known to have a good prognosis $[5,6]$. PTC is the most common histologic type of differentiated thyroid cancer and comprises $75-85 \%$ of all thyroid malignancies in the United States [3]. Although PTC is curable, it can dedifferentiate into a more aggressive and lethal thyroid cancer characterized by metastasis to the cervical lymph nodes (LNs) [6]. According to the American Thyroid Association (ATA), thyroid sonography of cervical LNs should be performed in patients with known or suspected thyroid nodules before patients undergo a thyroidectomy [7-9]. The structure of LN metastasis differs from that of normal $\mathrm{LN}$, which include cystic change, loss of normal architecture, calcification, and hyperechogenicity [10]. Because of its metastatic potential, intensive research has been done, and the diagnosis of thyroid cancer, particularly PTC, has increased immensely during the past three decades. Additionally, PTC recurs in approximately $30 \%$ of cases; therefore, it requires further molecular characterization.

Besides PTC, follicular thyroid cancer (FTC) is the other differentiated thyroid cancer $[11,12]$. The cells from follicular adenomas (FA) and follicular carcinomas appear identical by cytology assay; cancer is diagnosed only by identifying capsular or vascular invasion on histologic specimens [13]. According to the WHO Classification of Tumors of Endocrine Organs, FTC is defined as a malignant epithelial tumor showing follicular cell differentiation and lacking the diagnostic nuclear features of PTC. Because of the difficulty of definitively diagnosing FTC, there has been an overdiagnosis of the follicular variant of papillary thyroid cancer [11]. Since FTC cannot be detected by cytology, diagnostic thyroid surgery is required, which shows a $20 \%$ positive malignant lesion out of all these surgeries [4]. 
According to the pattern of invasion, there have been two forms of follicular thyroid cancer identified, the minimally invasive and the widely invasive carcinomas. Fewer data are available on FTC and FA, so the goal of this review is to provide information on reliably discriminating FA from FTC. Mutation analysis showed that more than 30\% of all FTC do not show any known mutations $[14,15]$; therefore, the discovery of additional molecular markers is useful for the improvement of presurgical diagnosis of FTCs.

Anaplastic thyroid cancer (ATC) is another type of thyroid cancer with extremely poor prognosis [16-18]. ATC is an aggressive undifferentiated tumor, and because of its aggressiveness, the American Joint Committee classifies this cancer as a stage IV regardless of its tumor size, nodal status, or the absence or presence of distant metastases [18]. Although ATC is the rarest type of thyroid cancer, these characteristics make it contribute to $50 \%$ of the mortality from thyroid cancer. ATC poses a significant clinical challenge as it is highly aggressive, and there are no effective therapeutic options. Messenger RNA (mRNA) expression profiling demonstrates that ATC has a distinctive molecular signature [19]. Therefore, this systematic review adds information about the candidate biomarkers that are frequently expressed in ATC and may be used as potential diagnostic/prognostic biomarkers and therapeutic targets.

Lastly, medullary thyroid cancer (MTC) occurs in two forms: hereditary (HMTC) and sporadic (SMTC). Out of all, $75 \%$ of MTCs are sporadic and typically present at a late stage with nodal metastasis present at the time of diagnosis and a relatively poor prognosis.

In the past several years, knowledge of the genetic alterations of thyroid tumors has greatly increased. There is a remarkable correlation between genotype and phenotype, indicating that distinct molecular changes are associated with stages in a multistep tumorigenic process. Alterations of genes or gene pathways are linked to specific histologic features. This genotype/phenotype correlation provides the rationale for the numerous studies on gene expression profiling. The analysis of mRNA expression features may represent a useful tool for the preoperative diagnosis of thyroid nodules [20].

The translation from basic science to the potential diagnostic application also extends to recent studies on microRNAs (miRNAs) [21, 22]. These are small noncoding RNA molecules with critical functions for cell differentiation that negatively regulate gene expression, including known oncogene and tumor suppressor genes. Different subtypes of thyroid cancer are associated with specific miRNA profiles [23-31]. Technological advances allow the analysis of formalin-fixed routinely processed samples [32] as well as fineneedle aspiration biopsy (FNA) specimens using highthroughput sequencing [33], and as such their use as diagnostic and prognostic markers appears very promising. In this report, we have outlined the miRNAs involved in different subtypes of thyroid cancer from the existing literature in a comprehensive manner so that we can distinguish the subtype-specific miRNA signatures and follow their roles in tumor aggressiveness in future studies.

\section{2. miRNAs}

When miRNAs were discovered over 30 years ago, it was believed that they were conventional protein-coding genes. Yet after experimentation, Hammond made the remarkable discovery that instead of coding for protein, they represented a new class of long regulatory noncoding RNA which was later called miRNA [31]. miRNAs are 19-24 nucleotides long and bind to the $3^{\prime}$ UTR in messenger RNA to inhibit functional gene production. Their importance is attributed to their participation in development, cell proliferation, differentiation, apoptosis, and their role in many diseases including cancer [34]. Data also show that miRNAs can be used as potential biomarkers for the diagnosis and prognosis of a variety of diseases such as cancer, neurological disorders, cardiovascular diseases, and type-II diabetes; they can not only be used to monitor treatments but also for patient stratification $[35,36]$.

Differential miRNA expression profiles have been reported in tumors and normal tissues; such a phenomenon suggests the key role of miRNA in tumorigenesis. A single miRNA can have multiple targets with varying binding strengths. In addition to the amount and availability of the binding site, the binding strength determines whether miRNAs inhibit gene production by inhibiting translation or stimulating degradation of mRNA. Weak binding strength tends to inhibit the gene expression by targeting translation. mRNA instability and degradation most likely result when the corresponding miRNA sequence is completely complimentary, leading to tight binding and activation of the RNA-mediated interference (RNAi) pathway [37].

\section{3. miRNAs and Cancer}

There have been many studies that have identified the links between changes in miRNA expression from normal to cancer cells and tumor growth. With these findings and various tumors, miRNAs are currently being tested as potential diagnostic or prognostic biomarkers [38-41]. The most striking evidence that links miRNAs with cancer is their large alteration in expression in malignant cells compared to benign cells. miRNA expression is dysregulated in human cancer through various mechanisms, such as amplification or deletion of miRNA genes, abnormal transcriptional control of miRNAs, epigenetic changes, and defects in the miRNA processing machinery. Because of this dysregulation, miRNAs are classified as oncogenes or tumor suppressor genes. It is important to note that miRNAs are not always classified as tumor suppressors or as oncogenes; their classification depends on the specific tissue or cell type context in which they are expressed [41]. These genes regulate different carcinogenic processes in various types of malignancies [42]. The dysregulated miRNAs have an influence on the hallmarks of cancer, including proliferative signaling, evading growth suppressors, resisting cell death, inducing angiogenesis, activating invasion, and metastasis. Recent studies in cancer have shown that miRNAs play a very important role in cell differentiation; they can regulate the transformation of cancer cells and the acquisition of the 
epithelial-mesenchymal transition phenotype which is greatly associated with drug resistance [40]. Cancers including chronic lymphocytic leukemia, colon cancer, glioblastoma, and astrocytoma are associated with a change in the level of miRNA expression [43-48].

\section{4. miRNA Expression Profile in Thyroid Cancer Subtypes}

Numerous studies are going on to use miRNAs as potential biomarkers for human thyroid cancer diagnosis, prognosis, and therapeutic targets; these need further validation using a large cohort of samples. miRNAs are highly specific for a given cell and disease, relatively stable due to its shorter length (24 nucleotides) and therefore can get detected and reliably measured in various biological materials, including archived formalin-fixed and paraffin embedded samples or serum. With the availability of high throughput next-generation sequencing, miRNAs can be detected more abundantly and differentially from control compared with other miRNA microarrays, northern blots, TaqMan microRNA Assays Human panel, and confirmed by qRT-PCR assays. The miRNA expression profile presents a significant variability between different kinds of thyroid cancers, even if they originate from the same type of thyroid cells [21, 49]. For example, C-cell-derived medullary thyroid carcinoma has a miRNA expression profile significantly different from those of thyroid tumors originating from follicular cells. Although papillary carcinomas, conventional follicular adenomas and carcinomas, and oncocytic follicular adenomas and carcinomas all are originating from follicular cells, they show different and distinct miRNA expression profiles [50]. Although the exact biological roles of miRNAs in thyroid carcinogenesis remain to be fully studied, the specific miRNA expression profile in thyroid tumors compared to normal thyroid tissue may be useful in the diagnosis and treatment of thyroid neoplasia. Moreover, the different miRNA expression patterns in different types of thyroid tumors could be useful for their classification, serving as diagnostic markers and therapeutic markers [50, 51]. Analysis of miRNA expression levels and detection of circulating miRNAs can be used for the early diagnosis of thyroid cancer and for monitoring treatment responses [51].

In this review, we focus on miRNA expression in thyroid cancer to evaluate histological type-specific miRNA signatures, functional interactions with mRNAs, and miRNAs with the best potential as prognostic or diagnostic markers. Our goal is to provide a resource to strategize what future studies need to be performed. Although there are other review papers about miRNA and thyroid cancer subtype, such as review articles by Marini et al. and Celano et al. $[50,51]$, our review is more comprehensive and easier to understand by using Venn diagram. Venn diagram helps to illustrate upregulated and downregulated different miRNAs profile in different thyroid cancer subtypes and highlight the miRNAs that are in common. Moreover, our review especially focuses on the let-7 family and their expression profile in different thyroid cancer subtypes, including anaplastic thyroid cancer, which is not focused on other review papers to our best knowledge.

\section{5. miRNAs in Papillary Thyroid Cancer}

As papillary thyroid carcinoma (PTC) is the most common thyroid cancer, most miRNA expression research has been done in PTC cell lines or patient samples $[23,24,26,28,32,52-64]$. A recent study showed the expression of miRNA-146b to be 28.9-fold higher in PTC tumors compared with control tissues [65]. Many other studies have found miR-146b expression upregulated in PTC $[23,28,32,57,58,66-68]$. Various studies have found multiple functions of miR-146 including its roles in migration, invasion, proliferation, cell cycle, and chemotherapy resistance in BRAF-mutated cells [68-70]. Specifically, Deng et al. [69] found the Zinc Ring Finger 3 (ZNRF3) gene to be a direct target of miR-146b-5p leading to the downregulation of the gene product, resulting in ell migration, invasion, and epithelial-to-mesenchymal transition (EMT). More importantly, clinically, a study identified miR-146 as a possible prognostic biomarker, as they demonstrated increased miR146 levels as an independent risk factor for worse prognosis in PTC patients [68].

$\mathrm{Ab}$ Mutalib et al. found miR-146b expression is significantly higher in PTC patients with lymph node metastasis positive (PTC LNM-P) or PTC patients with lymph node-negative (PTC LNN) fold change 6.0 and 4.7, respectively, when compared with adjacent normal thyroid tissue [71]. Additionally, other studies also found the miR146b expression is greater in PTC LNM-P than in PTC LNN $[52,53,56,69]$. Another miRNA that differed significantly in expression in PTC LNM-P compared with PTC LNN was miR-204. This miRNA was downregulated significantly in PTC LNM-P patients [71]. Additionally, miR-204 expression is downregulated in PTC versus adjacent normal thyroid tissue [24]. The miR-204, classified as a tumor suppressor miRNA, has been found to be downregulated in other cancers such as renal carcinoma [44], cervical cancer [45], and breast cancer [46]. Furthermore, in other cancer types, downregulated miR-204-5p has potential for use as a prognostic biomarker [47, 48]. As a tumor suppressor miRNA, Liu et al. found miR-204 in PTC cell lines inhibits cell proliferation, induces apoptosis, and arrests the cell cycle; additionally, miR-204 inhibited tumorigenicity in vivo [72].

Acibucu et al. and Yip et al. also found upregulation of miRNA 221 and miRNA 222 in PTC compared with normal thyroid tissue and in PTC with an increased risk of recurrence [56, 57]. Therefore, the more miRNA 146b, 221, and 222 are expressed, the higher the risk of recurrence of PTC because patients with upregulated expression of these miRNAs also had PTC that manifests with capsule invasion, vascular invasion, or lymph node metastasis. Of these 3 miRNAs, miRNA 146b was found to have a statistically significant difference in PTC with capsule invasion, vascular invasion, and lymph node metastasis. Similar to miRNA 146b, miRNA 221 and miRNA 222 are also involved in cell proliferation in thyroid cancer [23], and most notably 
upregulation of these miRNAs corresponds to increased tumor aggression [49]. In addition to normal thyroid tissue, another study found that miRNA 221 and 222 are upregulated in PTC compared with follicular adenoma and hyperplastic nodules $[26,66]$. In agreement with Dai et al. also found miRNA 221 to be an independent risk factor for PTC recurrence [73].

Cantara et al. demonstrated that miR-95 and miR-190 are accurate and sensitive diagnostic markers for PTC in Caucasians [74]. Their results showed that miR-95 has a sensitivity of $94.9 \%$ and specificity of $98.7 \%$ in distinguishing PTC from nodular goiters and normal thyroid tissue. The sensitivity increases to $100 \%$ when miR-95 is combined with miR-190, which also has high diagnostic power. However, as this study only evaluated the diagnostic power of these two miRNAs in PTC, their diagnostic value can decrease depending on the expression pattern of miR-95 in other thyroid cancer types.

Another study [75] showed that miR-654-3p expression decreased in PTC over a long period of progression in vivo, which inversely correlated with EMT. The study then went on to demonstrate in vitro the tumor suppressor functions of miR-654-3p such as an increase in apoptosis and a decrease in cell proliferation, which has also been reported in prostate cancer cell lines [76]. Upon profiling serum miRNA in search of a serum biomarker for PTC, a pilot study [64] found 3 miRNAs that had statistically significant fold changes (5-fold change or more) between benign thyroid masses and PTC. One of the miRNAs was downregulated, miR-199b-3p, and the others were upregulated, let7b-5p and miR-10a-5p. According to Mohamad et al.'s study miR-205 is upregulated for PTC and miR-205 is associated with angiogenesis, proliferation, and invasion [77]. miRNA signature in PTC reported in the literature is shown in Table 1.

\section{6. miRNAs in Follicular Thyroid Cancer}

Besides PTC, follicular thyroid cancer (FTC) is the other type of differentiated thyroid cancer. In contrast to papillary thyroid cancer, follicular adenomas and carcinomas share the same tumorigenic pathway. Many studies have shown that the mRNA/miRNA expression profiles of follicular neoplasms differ from those of papillary carcinoma [86]; however, molecular signatures that clearly distinguish between follicular adenoma and follicular carcinoma need further validation in large studies. In a search for biomarkers to distinguish follicular thyroid cancer and PTC, dysregulated miRNA expression is well documented in follicular neoplasms. Samsonov et al. [86] found miRNA-21 and miRNA-181a-5p, which are exosomal miRNA, can differentiate the two cancer types with a sensitivity of $100 \%$ and specificity of $77 \%$. Expression of miR-197 and miR-346 is significantly higher in follicular carcinoma than in follicular adenoma [21, 25, 27, 29, 49, 87].

In a meta-analysis study, miR-637, miR-181c-3p, miR206, and miR-7-5p were revealed to be de novo potential FTC biomarkers; especially miR-7-5p showed the potential to distinguish between benign and malignant thyroid tissue in several published datasets [27]. Stokowy et al. [29] found two miRNA classifiers consisting of miR-484 and miR-148b$3 \mathrm{p}$ and distinguishes mutation-negative FTC from follicular thyroid adenomas with a sensitivity and specificity of $89 \%$ and $87 \%$, respectively. In another study, Stokowy et al. [88] revealed different two miRNA classifiers consisting miR-7$5 p$ and miR-7-2-3p for distinguishing PTCs and FTCs from benign thyroid masses. These two miRNA classifiers yielded a sensitivity and specificity of $82 \%$ and $49 \%$, respectively.

Besides the miRNAs reported above, one study found that miR-146b, miR-183, and miR-221 (which are observed to be deregulated in PTC) are upregulated and miR-199b was downregulated in FTC versus normal thyroid cells [25].

Follicular variant of papillary thyroid carcinoma is the most common variant of PTC and is diagnosed in about 15-30\% of PTC, sharing features of PTC and FTC on clinical, morphological, and genetic levels. It is important to distinguish between FTC and PTC with the follicular variant in clinical practice. Although miRNA deregulation was extensively studied in PTCs and FTCs, limited information is available for PTC with the follicular variant. Despite the high similarity in miRNA expression between PTC with the follicular variant and classical PTC, Dettmer et al. demonstrated that miR-375 is highly upregulated in PTC with the follicular variant and to a lesser extent in classic PTC, but not in FTC, hyperplastic nodule, or normal thyroid [30]. Besides, miR-181a-2-3p and miR-99b-3p can predict relapse-free survival in patients with PTC with follicular variant, thus may potentially provide important diagnostic and predictive value. miRNA signature in FTC reported in the literature is shown in Table 2 .

\section{7. miRNAs in Anaplastic Thyroid Cancer}

Anaplastic thyroid carcinoma (ATC) is a highly malignant tumor. ATC is not beneficial in the radioactive scan but present with high mitotic activity in histologic appearance. miRNA profile showed significant downregulation of miR30d, miR-30a-5p, miR-125b, and miR-26a expression. Out of these, a possible pathogenic role is suspected for miR125b and miR-26a [91]. As seen in studies with PTC and FTC, miR-222 is deregulated in ATC. While miR-222 is upregulated in ATC, miR-25 is found to be downregulated in ATC cell lines compared with benign thyroid cells [92]. miR-30a and miR-200, two tumor suppressor miRNAs, are downregulated in ATC compared with the differentiated thyroid tumors and normal thyroid tissue [93, 94]. The downregulation of these miRNAs correlates with advanced tumor differentiation and increased tumor aggressiveness and mortality [93]. EMT has been implicated in ATC, and miR-30a normally inhibits invasion, migration, EMT markers, and lysyl oxidase (LOX) expression. Specifically, LOX inhibits cellular division, invasion, and migration in vitro and in vivo, demonstrating the potential for the miR30a-LOX-axis as a target for ATC treatment [93]. The miR200 family was recently identified as a suppressor of EMT and studies indicate a correlation between the downregulation of this miRNA family and the invasive potential of ATCs $[95,96]$. The miR-200 family controls expression of essential EMT/mesenchymal-epithelial transition (MET) 
TABLE 1: Up and downregulated miRNAs in papillary thyroid cancer.

\begin{tabular}{|c|c|c|c|}
\hline \multicolumn{4}{|c|}{ Papillary thyroid cancer } \\
\hline Upregulated miRNA & Ref & Downregulated miRNA & Ref \\
\hline $\begin{array}{l}\text { miR-146b, miR-221, miR-222, miR- } \\
21^{*}, \mathrm{miR}-181 \mathrm{a} / \mathrm{c}^{*}, \mathrm{miR}-155, \mathrm{miR}- \\
\text { 181b, miR-221, miR-224, let7b-5p, } \\
\text { miR-10a-5p, miR-31, miR-122a, } \\
\text { miR-146b, miR-155, miR-187, miR- } \\
\text { 205, miR-146b-3p, miR-146-5p, } \\
\text { miR-222-5p, miR-181a, miR-551b- } \\
\text { 3p, miR-145, miR-182, miR-146b- } \\
\text { 5p, miR-25-3p, miR-451a, miR-140- } \\
\text { 3p, let7i }\end{array}$ & $\begin{array}{l}{[21,23,24,28,30,49,53-57} \\
62,64,67,68,78-84]\end{array}$ & 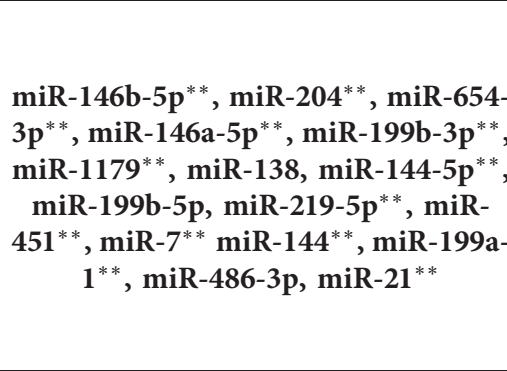 & $\begin{array}{c}{[24,30,52,68,72,75} \\
78,82,83,85]\end{array}$ \\
\hline
\end{tabular}

${ }^{*}$ miRNA targets genes by miRNA target screen: only specific upregulated miRNA and their target genes links in papillary thyroid cancer are underlined. http://www.mirdb.org/cgi-bin/search.cgi?searchType=miRNA\&searchBox=hsa-miR-21-5p\&full=1. http://www.mirdb.org/cgi-bin/search.cgi? searchType $=$ miRNA\&searchBox $=$ hsa $-\mathrm{miR}-181 \mathrm{a}-5 \mathrm{p} \&$ full=1. http://www.mirdb.org/cgi-bin/search.cgi? searchType=miRNA\&searchBox=hsa-miR-181c5p\&full=1. http://www.mirdb.org/cgi-bin/search.cgi?searchType=miRNA\&searchBox=hsa-miR-181b-5p\&full=1. http://www.mirdb.org/cgi-bin/search.cgi? searchType=miRNA\&searchBox=hsa-let-7b-5p\&full=1. http://www.mirdb.org/cgi-bin/search.cgi?searchType=miRNA\&searchBox=hsa-miR-10a-5p\&full=1 . http://www.mirdb.org/cgi-bin/search.cgi?searchType=miRNA\&searchBox=hsa-miR-31-5p\&full=1. http://www.mirdb.org/cgi-bin/search.cgi? search Type $=$ miRNA\&searchBox $=$ hsa-miR-146a $-5 \mathrm{p} \&$ full=1. http:/www.mirdb.org/cgi-bin/search.cgi? searchType=miRNA\&searchBox=hsa-miR-2225p\&full=1. http://www.mirdb.org/cgi-bin/search.cgi?searchType=miRNA\&searchBox=hsa-miR-551b-3p\&full=1. http://www.mirdb.org/cgi-bin/search.cgi? searchType=miRNA\&searchBox=hsa-miR-145-5p\&full=1. http://www.mirdb.org/cgi-bin/search.cgi?searchType=miRNA\&searchBox=hsa-miR-182-5p\&full=1. http://www.mirdb.org/cgi-bin/search.cgi?searchType=miRNA\&searchBox=hsa-miR-146b-5p\&full=1. http://www.mirdb.org/cgi-bin/search.cgi? searchType=miRNA\&searchBox=hsa-miR-25-3p\&full=1. http://www.mirdb.org/cgi-bin/search.cgi?searchType=miRNA\&searchBox=hsa-miR-451a\&full=1. http://www.mirdb.org/cgi-bin/search.cgi?searchType=miRNA\&searchBox=hsa-miR-140-3p\&full=1. http://www.mirdb.org/cgi-bin/search.cgi? searchType $=$ miRNA\&searchBox $=$ hsa-let-7i-5p\&full $=1 .{ }^{* *}$ miRNA targets genes by miRNA target screen: only specific downregulated miRNA and their target genes links in papillary thyroid cancer are underlined. http://www.mirdb.org/cgi-bin/search.cgi?searchType=miRNA\&searchBox=hsa-miR-146b5p\&full=1. http://www.mirdb.org/cgi-bin/search.cgi?searchType=miRNA\&searchBox=hsa-miR-204-5p\&full=1. http://www.mirdb.org/cgi-bin/search.cgi? searchType=miRNA\&searchBox=hsa-miR-654-3p\&full=1. http://www.mirdb.org/cgi-bin/search.cgi? searchType=miRNA\&searchBox=hsa-miR146a-5p\&full=1. http://www.mirdb.org/cgi-bin/search.cgi?searchType=miRNA\&searchBox=hsa-miR-199b-3p\&full=1. http://www.mirdb.org/cgi-bin/ search.cgi? searchType=miRNA\&searchBox=hsa-miR-1179\&full=1. http://www.mirdb.org/cgi-bin/search.cgi?searchType=miRNA\&searchBox=hsa-miR-1445p\&full=1. http://www.mirdb.org/cgi-bin/search.cgi?searchType=miRNA\&searchBox=hsa-miR-219a-5p\&full=1. http://www.mirdb.org/cgi-bin/search.cgi? searchType=miRNA\&searchBox=hsa-miR-451a\&full=1. http://www.mirdb.org/cgi-bin/search.cgi? searchType=miRNA\&searchBox=hsa-miR-7-5p\&full=1. http://www.mirdb.org/cgi-bin/search.cgi? searchType=miRNA\&searchBox=hsa-miR-144-3p\&full=1. http://www.mirdb.org/cgi-bin/search.cgi? searchType=miRNA\&searchBox=hsa-miR-199a $-5 \mathrm{p} \&$ full $=1$. http $/ /$ www.mirdb.org $/$ cgi-bin $/$ search. cgi searchType=miRNA\&searchBox=hsa $-\mathrm{miR}-$ $21-5 \mathrm{p} \&$ full $=1$.

modulators, namely, ZEB1/2, SNAI2, and TGF $\beta 2$, thus it is suggested to interfere with metastasis by sustaining an epithelial character of tissues [95-97].

Similar to miR-30a, miR-125b which is also a tumor suppressor miRNA, is downregulated in ATC. Another study suggests that the downregulation of miR-12b leads to PIK3CD upregulation, which is known to be involved in cell survival, growth, division, and glucose homeostasis [94, 98].

Family genes of let-7 are located on different chromosomes and are abundantly expressed in the normal thyroid gland (let-7a, let-7b, let-7c, let-7d, let-7e, let-7f, and let-7g) [99]. Deregulation of let-7 is observed in several types of cancer, and its tumor suppressor effects are usually abolished by its downregulation. Let-7 was found to be downregulated in ATC [94]. Downregulation of several members of the let-7 family is observed in well-differentiated thyroid cancer (PTC and FTC) [23, 24, 89, 90, 99], but a marked decrease in the expression of let-7a, let-7c, let-7d, let-7f, let-7g, and let-7i is also observed in ATC $[26,91,100]$.

Fassina et al. [101] found that a 4-miRNA signature consisting of miR-26a, miR-146b, miR-221, and miR-222, is able to distinguish primary lymphomas from ATC with a sensitivity greater than $80 \%$ and a specificity greater than 90\%. Clusters of miR 17-92 were found in ATC cells [102]. The upregulation of miR-20a was shown in ATC [103].
Upregulation of miR-146b, miR-221, and miR-222 and downregulation of miR-200 and miR-30 were reported [104]. Experimentally, it was shown that antisense-miR-21 enhances differentiation/apoptosis and reduces cancer stemness in ATC [105]. Mechanistically, it was shown that miR-99a inhibited tumorigenesis through targeting mTOR in ATC; on the other hand, miR-20a upregulation targeted LIMK1 in ATC. Therefore, strategy to restore tumor suppressor miRNAs and suppress the oncogenic miRNA will be a very promising therapeutic approach to ameliorate the treatment of ATC. miRNA signature in ATC reported in the literature is highlighted in Table 3.

\section{8. miRNAs in Medullary Thyroid Cancer}

In a miRNA microarray expression profile of a primary cohort of 12 SMTC and 7 HMTC samples, a number of miRNAs were identified to be differentially expressed $[106,107]$. It was shown that overexpression of miR-183 and 375 in all MTCs predicted lateral lymph node metastases, residual locoregional disease, and mortality [106]. Pennelli et al. showed that miR-224 upregulation represents a prognostic biomarker associated with a better outcome in MTC patients [108]. Another group showed that, by array analysis, miR-200b and miR-200c are significantly 
TABLE 2: Up- and downregulated miRNAs in follicular thyroid cancer.

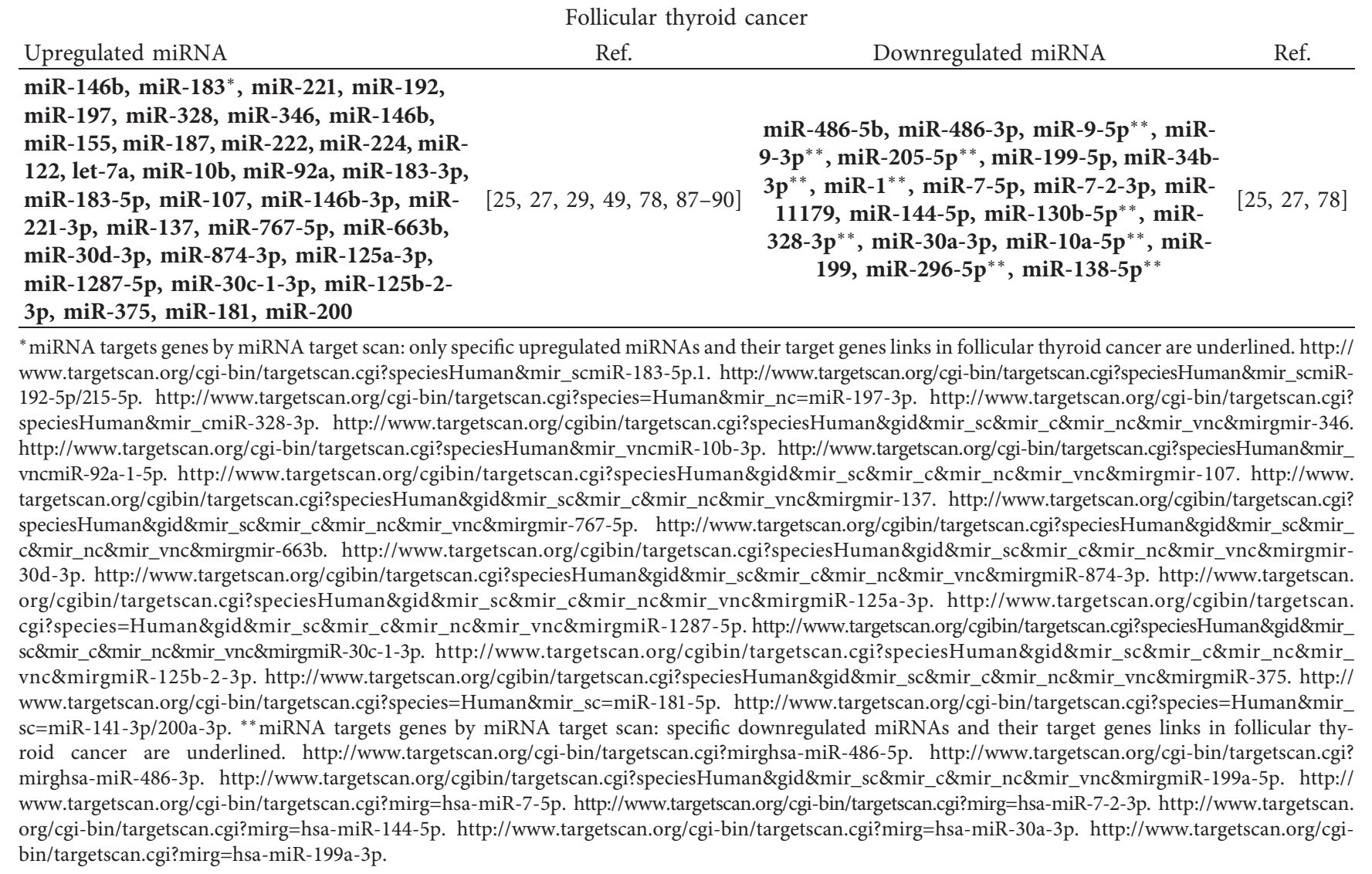

downregulated in MTC metastases, consistent with reports showing downregulation of class of this miRNA during the metastatic process [109]. Hudson et al. reported that miR375 and miR-10a were overexpressed, whereas miR-455 was underexpressed in MTC [110].

Chu et al. studied miR-21 and concluded that overexpression of miR-21 and MALAT1 regulate MTC progression and miR-21 inhibition reduced cell proliferation [111]. As mentioned above, many researchers have made it their mission to find the markers of metastatic cells and aggressive tumor behavior. Santarpia et al., went further to uncover 10 markers, two of which have been mentioned before, miR10a, miR-200b/-200c, miR-7, and miR-29c which were downregulated, whereas miR-130a, miR-138, miR-193a-3p, miR-373, and miR-498 were upregulated in MTC (or MTC progression?). These markers were involved in MTC pathways [109]. miRNA signature in MTC reported in the literature is depicted in Table 4.

\section{Potential Therapeutic Implication of Targeting miRNA in Thyroid Cancer}

miRNAs play a critical role in the regulation of various diseases, including thyroid cancer. Because the dysregulation of miRNA can be seen in many diseases, the possibility of using these as therapeutic agents has increased. According to Christopher et al., different laboratories have conducted recent studies in humans and animals. Their study shows us that miRNAs could become established as a new kind of drug [113]. Characteristics such as size and their conserved and known sequences make miRNAs attractive in terms of drug development. The effect that upregulated miRNA has in our biology can be eliminated by utilizing inhibitors or anti-miR. These are considered synthetic molecules that are used to prevent the miRNA from binding to its target. On the contrary, when miRNA levels are downregulated, it can be solved by targeting its positive regulation. An example of how this can be achieved is by delivering synthetic miRNA that imitates mature miRNAs. One of the approaches used to correct this anomalous miRNA expression is by synthesizing antisense oligonucleotides with a complementary sequence. These have been used to suppress dysfunctional mRNAs for more than four decades. It is widely recognized that the activation of oncogenes can lead to the formation of cancer, and to prevent this, artificial antisense miRNAs can be synthesized. By synthesizing this antisense oligonucleotide, it could be directed to block the miRNA that is causing cancer (known as oncomirs). However, vast knowledge must be known to use this technology. This includes the identity of the miRNAs that are dysregulated, their mechanism of action, applicability by RNAi, delivery of miRNAs, and their active form in vivo [113].

Another method that can be used to approach the therapeutic targeting of miRNAs is by expression vectors or miRNA sponges. This vector-based plan of action consists of 
TABLE 3: Up- and downregulated miRNAs in anaplastic thyroid cancer.

\begin{tabular}{|c|c|c|c|}
\hline \multicolumn{4}{|c|}{ Anaplastic thyroid cancer } \\
\hline Upregulated miRNA & Ref. & Downregulated miRNA & Ref. \\
\hline $\begin{array}{l}\text { miR-222, miR-137, miR-155, miR-187, miR- } \\
\text { 205, miR-214*, miR-221, miR-224, miR-302*, } \\
\text { miR-17* }\end{array}$ & {$[49,78,91,92]$} & $\begin{array}{c}\text { miR-25**, miR-26a**, miR-30a-5p, miR-30d, } \\
\text { miR-125**, let-7, miR-29**, miR-30, miR-125b, } \\
\text { miR-12b }\end{array}$ & {$[78,91-93,98]$} \\
\hline
\end{tabular}

* miRNA targets genes by miRNA target scan: only specific upregulated miRNAs and their target genes links in anaplastic thyroid cancer are underlined. http://www.targetscan.org/cgi-bin/targetscan.cgi?species=Human\&mir_sc=miR-214-5p. http://www.targetscan.org/cgi-bin/targetscan.cgi?species=Human\&mir_ sc=miR-302-3p/372-3p/373-3p/520-3p. http://www.targetscan.org/cgi-bin/targetscan.cgi? species=Human\&mir_sc=miR-17-5p/20-5p/93-5p/106-5p/5193 p. ${ }^{* *}$ miRNA targets genes by miRNA target scan: specific downregulated miRNAs and their target genes links in anaplastic thyroid cancer are underlined. http://www.targetscan.org/cgi-bin/targetscan.cgi?species=Human\&mir_sc=miR-25-3p/32-5p/92-3p/363-3p/367-3p. http://www.targetscan.org/cgibin/targetscan.cgi?species=Human\&mir_sc=miR-26-5p. http://www.targetscan.org/cgi-bin/targetscan.cgi?species=Human\&mir_sc=miR-125-5p. http:// www.targetscan.org/cgi-bin/targetscan.cgi?species=Human\&mir_vnc=miR-125b-1-3p. http://www.targetscan.org/cgi-bin/targetscan.cgi?species=Human\&mir_ $\mathrm{sc}=\mathrm{miR}-29-3 \mathrm{p}$

TABLE 4: Up- and downregulated miRNAs in medullary thyroid cancer.

\begin{tabular}{|c|c|c|c|}
\hline \multicolumn{4}{|c|}{ Medullary thyroid cancer } \\
\hline Upregulated miRNA & Ref. & Downregulated miRNA & Ref. \\
\hline $\begin{array}{l}\text { miR-224, miR-375, miR-10a, miR-130a, miR-138, miR- } \\
\text { 193a-3p, miR-373, miR-1 }\end{array}$ & {$[107-110,112]$} & 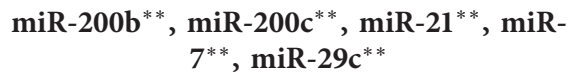 & {$[109,111]$} \\
\hline
\end{tabular}

*miRNA targets genes by miRNA target scan: only specific upregulated miRNAs and their target genes links in medullary thyroid cancer are underlined. http://www.targetscan.org/cgi-bin/targetscan/vert_71/targetscan.cgi?mirg=hsa-miR-224-5p. http://www.targetscan.org/cgi-bin/targetscan/vert_71/ targetscan.cgi?mirg=hsa-miR-375. http://www.targetscan.org/cgi-bin/targetscan/vert_71/targetscan.cgi?mirg=hsa-miR-10a-5p. http://www.targetscan.org/ cgi-bin/targetscan/vert_71/targetscan.cgi?mirg=hsa-miR-130a-5p. http://www.targetscan.org/cgi-bin/targetscan/vert_71/targetscan.cgi?mirg=hsa-miR-1385p. http://www.targetscan.org/cgi-bin/targetscan/vert_71/targetscan.cgi?mirg=hsa-miR-193a-3p. http://www.targetscan.org/cgi-bin/targetscan/vert_71/ targetscan.cgi?mirg=hsa-miR-373-5p. http://www.targetscan.org/cgi-bin/targetscan/vert_71/targetscan.cgi?mirg=hsa-miR-1-5p. ${ }^{* *}$ miRNA targets genes by miRNA target scan: specific downregulated miRNAs and their target genes links in anaplastic thyroid cancer are underlined. http://www.targetscan.org/cgibin/targetscan/vert_71/targetscan.cgi?mirg=hsa-miR-200b-5p. http://www.targetscan.org/cgi-bin/targetscan/vert_71/targetscan.cgi?mirg=hsa-miR200c-5p. http://www.targetscan.org/cgi-bin/targetscan/vert_71/targetscan.cgi?mirg=hsa-miR-21-5p. http://www.targetscan.org/cgi-bin/targetscan/vert_71/ targetscan.cgi?mirg=hsa-miR-7-2-3p. http://www.targetscan.org/cgi-bin/targetscan/vert_71/targetscan.cgi?mirg=hsa-miR-29c-5p.

introducing an mRNA that contains many binding sites for the specific miRNA that is dysregulated. Hence why it is considered a sponge. By overexpressing these mRNA-specific sponges, the miRNA that is causing the oncogenic or tumor-suppressive role binds to the specific sites in the sponge, thus liberating the target mRNAs and allowing their expression. Although many studies of this approach have been performed in vitro, only transgenic animals have been used for studies in vivo. The last approach taken in the therapeutic targeting of miRNAs is the usage of smallmolecule inhibitors. According to Li and Rana these approaches utilize reporter-based assay systems for compound library screening, and they have identified small molecules that could specifically inhibit miRNA expression [114]. Instead of inhibiting target recognition, this approach focuses on the transcriptional regulation of these targeted miRNAs. However, the study specifies that the downfall of this approach is its high $\mathrm{EC}_{50}$ values (effector concentration for half-maximum response) and the lack of information on direct targets.

According to Wojcicka et al., no study has reported the clinical use of miRNAs for thyroid cancer treatment, although its strong potential has been mentioned in many functional and preclinical studies [115]. Most of these studies focus on PTC as it is the most common thyroid cancer. However, few to no studies focus on the potential of targeting miRNA on FTC and PDTC. The article specifies that miRNAs not only affect the regulation of tumor growth but they also influence the results of other therapies for thyroid cancer. Often, patients are not obtaining the desired results from the radioiodine therapies due to the lowered expression and function of the $\mathrm{Na}^{+} / \mathrm{I}^{-}$symporter (NIS). It was discovered that the overexpression of miR-339 and miR$146 \mathrm{~b}$ reduced the expression NIS in thyroid tumors. In contrast, the inhibition of these miRNAs allowed thyroid cancer cells to increase the uptake of radioactive iodine.

Various biotech and pharmaceutical companies have already started to develop products that use miRNAs therapeutics. However, they are focusing on miRNA mimics and antagomiRs. We have already established that miRNA mimics are used to re-establish the concentration of the specific miRNA that is downregulated and that is causing the pathology. In change, antagomiRs are used to repress those miRNAs that are upregulated and that are causing the pathology. According to Bonneau et al., there are two challenges that these companies have faced that must be managed for the success of this novel project [116]. Those are the stability and the delivery of the treatment. Contrary to DNA, RNA molecules are very unstable because they contain a $2^{\prime}-\mathrm{OH}$ chemical group. The other challenge, the delivery of RNAs to the targeted organs is necessary as it maintains the specificity of the treatment. Passive strategies such as the usage of the liver, the spleen, and the lymph nodes are used to internalize accumulated particles. Another 


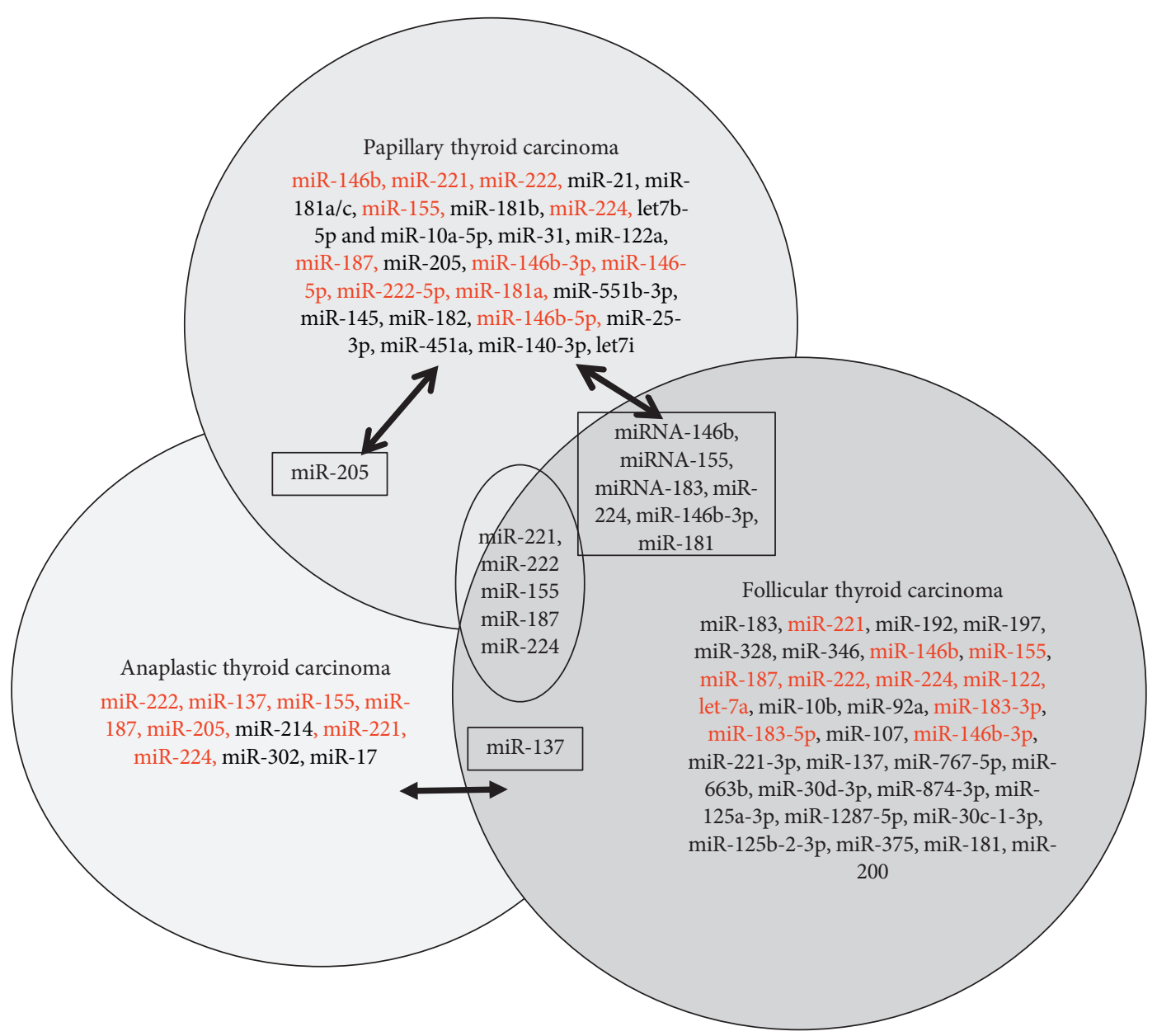

(a)

Figure 1: Continued. 


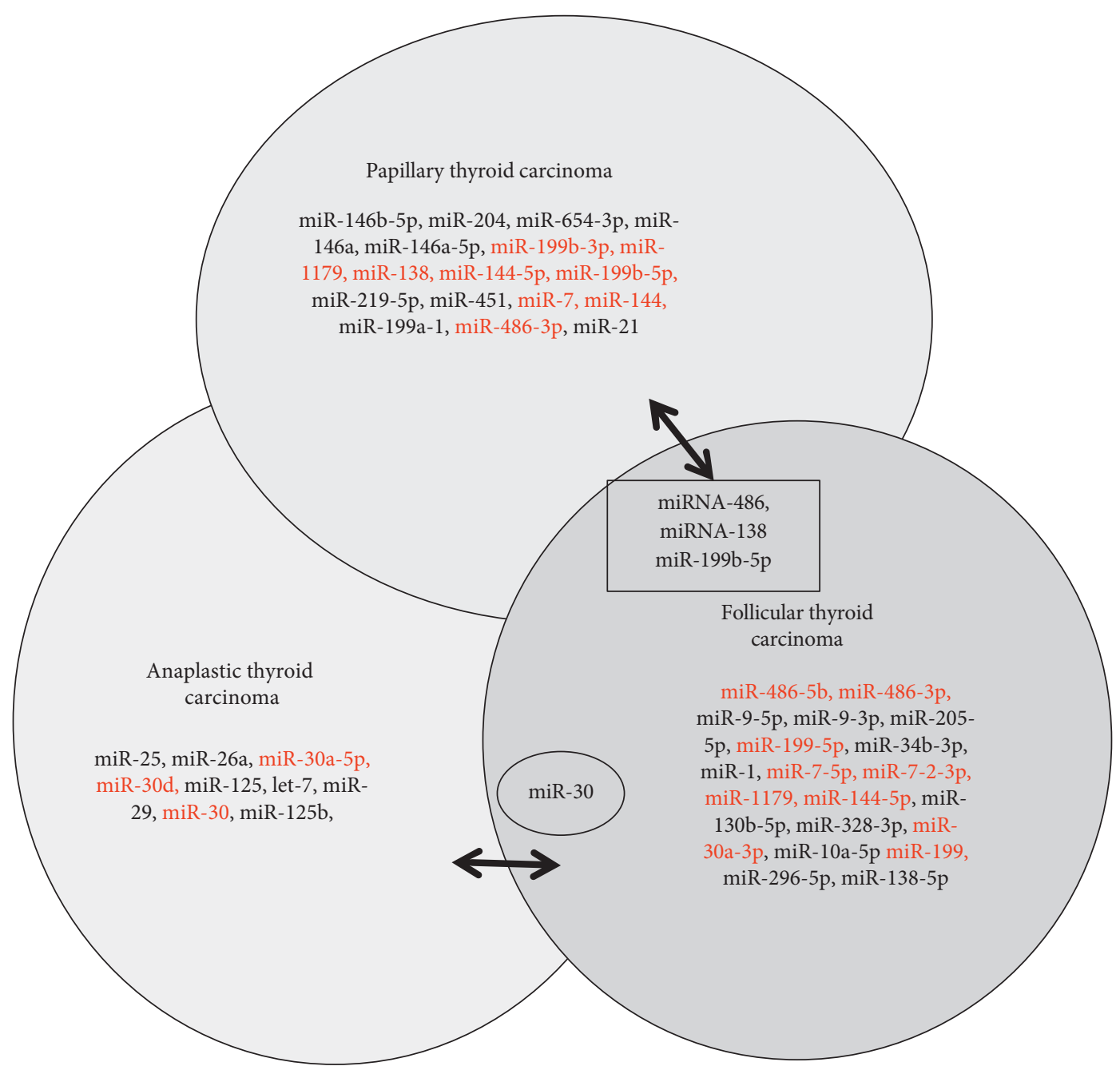

(b)

FIgURE 1: (a) Upregulated miRNAs. (b) Downregulated miRNAs.

strategy that could be used to deliver the therapy is through the active strategy. Here, the RNA or the particle with the specific molecule that will bind to the cell of interest will be endocytosed. There are no studies that elucidate the most favorable approach for thyroid cancer.

\section{Conclusions}

In conclusion, we have summarized our literature search data in a Venn diagram to show the upregulated and downregulated gene pulls in subtypes of thyroid cancer (Figures 1(a) and 1(b)). We have also found very few common miRNAs that were upregulated/downregulated as shown in Figure 1. Most of the upregulated/downregulated miRNAs were subtype-specific, out of which miR-221, miR222, miR-224, miR-155, and miR-187 were shown commonly in PTC, FTC, and ATC subtypes. Some of the miRNAs were common between PTC, and FTC, whereas very few overlapped between PTC to ATC (miR-205) and ATC to FTC (miR-137). However, we are not clear about the overlapping miRNAs or the subtype-specific miRNAs are important. Further studies need to clarify their roles.

With miR-146 being one of the most studied and most promising miRNAs involved with thyroid cancer, there is much interest in it as a gene target in hopes to reveal new prognostic biomarkers in PTC, especially those responsible for lymph node metastasis. Currently, more work is being done to profile miRNA in serum to find potential prognostic and diagnostic biomarkers. We suggest the need for more studies in vivo and multiple miRNA classifiers. Different studies have reported that high-throughput sequencing yielded more insight and better results in clarifying the relationship between miRNA and thyroid cancer $[20,24,33]$. The miRNA expression pattern is associated with tumor type, grade, and clinical outcomes. However, more efforts are still needed to screen miRNAs by deep sequencing in a large cohort of patient samples, especially for FTC and ATC. Though PTC is the most commonly diagnosed thyroid cancer, miRNA expression in ATC should be more thoroughly studied due to its large contribution to the mortality rate of thyroid cancers. 
The limitation of our review article is that we did not perform meta-analysis; therefore, this lacks quantitative or qualitative analyses due to the limited number of literature on miRNA for each subtype of thyroid cancer and thus could not reveal the biases, strengths, and weaknesses of existing studies cited here. We also did not provide the mechanism of up and downregulation of miRNA-clusters and their pathways linked to each subtype-specific mRNA target. That would lead to another extended review on each subtypespecific pathway analysis, which awaits further ongoing extensive research.

Future studies are needed not only to identify novel miRNAs, but also their biological functions, roles in tumorigenesis, and ability to predict thyroid cancer prognosis, diagnosis, and treatment. In order to plan promising therapeutic strategies, it is necessary to investigate both upregulated and downregulated pulls of miRNA signatures and their mRNA targets in each of the thyroid cancer subtypes.

Recently, our lab focused on the miRNA signature in different ethnicities. However, in the literature search, we found that very few studies have been conducted to demonstrate the ethnic differences in miRNA expression in thyroid cancer health disparities [58, 74, 117]. Our lab has found a family of interesting miRNA signatures in Asian Americans with implications for thyroid cancer health disparities (unpublished data). Further studies are needed to demonstrate differential expression patterns and their mRNA targets to stratify the stress-induced mechanisms in cancer health disparities.

\section{Conflicts of Interest}

The authors declare that they have no conflicts of interest.

\section{Acknowledgments}

The authors would like to thank Dr. Alfred A. Simental, Chair of the Department of Otolaryngology, Dr. Marino De Leon, Director, Center for Health Disparities and Molecular Medicine, Loma Linda University School of Medicine, for financial help. The authors like to thank Eunice Nyasani for her technical assistance in the preparation of the manuscript.

\section{References}

[1] T. Carling and R. Udelsman, "Thyroid cancer," Annual Review of Medicine, vol. 65, no. 1, pp. 125-137, 2014.

[2] R. L. Siegel, K. D. Miller, and A. Jemal, "Cancer statistics, 2019," CA: A Cancer Journal for Clinicians, vol. 69, no. 1, pp. 7-34, 2019.

[3] S. I. Sherman, P. Angelos, D. W. Ball et al., "Thyroid carcinoma," Journal of the National Comprehensive Cancer Network: JNCCN, vol. 5, no. 6, pp. 568-621, 2007.

[4] Z. W. Baloch, V. A. LiVolsi, S. L. Asa et al., "Diagnostic terminology and morphologic criteria for cytologic diagnosis of thyroid lesions: a synopsis of the national cancer institute thyroid fine-needle aspiration state of the science conference," Diagnostic Cytopathology, vol. 36, no. 6, pp. 425-437, 2008.
[5] Y. Ito, M. Fukushima, C. Tomoda et al., "Prognosis of patients with papillary thyroid carcinoma having clinically apparent metastasis to the lateral compartment," Endocrine Journal, vol. 56, no. 6, pp. 759-766, 2009.

[6] Y. J. Choi, J. S. Yun, S. H. Kook, E. C. Jung, and Y. L. Park, "Clinical and imaging assessment of cervical lymph node metastasis in papillary thyroid carcinomas," World Journal of Surgery, vol. 34, no. 7, pp. 1494-1499, 2010.

[7] E. K. Alexander, G. C. Kennedy, Z. W. Baloch et al., "Preoperative diagnosis of benign thyroid nodules with indeterminate cytology," New England Journal of Medicine, vol. 367, no. 8, pp. 705-715, 2012.

[8] D. S. Cooper, G. M. Doherty, B. R. Haugen et al., "Revised american thyroid association management guidelines for patients with thyroid nodules and differentiated thyroid cancer," Thyroid, vol. 19, no. 11, pp. 1167-1214, 2009.

[9] B. R. Haugen, A. M. Sawka, E. K. Alexander et al., "American thyroid association guidelines on the management of thyroid nodules and differentiated thyroid cancer task force review and recommendation on the proposed renaming of encapsulated follicular variant papillary thyroid carcinoma without invasion to noninvasive follicular thyroid neoplasm with papillary-like nuclear features," Thyroid, vol. 27, no. 4, pp. 481-483, 2017.

[10] A. C. Nachiappan, Z. A. Metwalli, B. S. Hailey, R. A. Patel, M. L. Ostrowski, and D. M. Wynne, "The thyroid: review of imaging features and biopsy techniques with radiologicpathologic correlation," RadioGraphics, vol. 34, no. 2, pp. 276-293, 2014.

[11] M. J. Schlumberger and M. Torlantano, "Papillary and follicular thyroid carcinoma," Best Practice and Research Clinical Endocrinology \& Metabolism, vol. 14, no. 4, pp. 601-613, 2000.

[12] I. S. Nam-Goong, H. Y. Kim, G. Gong et al., "Ultrasonography-guided fine-needle aspiration of thyroid incidentaloma: correlation with pathological findings," Clinical Endocrinology, vol. 60, no. 1, pp. 21-28, 2004.

[13] K.-C. Loh, F. S. Greenspan, L. Gee, T. R. Miller, and P. P. B. Yeo, "Pathological tumor-node-metastasis (pTNM) staging for papillary and follicular thyroid carcinomas: a retrospective analysis of 700 patients," The Journal of Clinical Endocrinology and Metabolism, vol. 82, no. 11, pp. 35533562, 1997.

[14] Y. E. Nikiforov, N. P. Ohori, S. P. Hodak et al., "Impact of mutational testing on the diagnosis and management of patients with cytologically indeterminate thyroid nodules: a prospective analysis of 1056 FNA samples," The Journal of Clinical Endocrinology \& Metabolism, vol. 96, no. 11, pp. 3390-3397, 2011.

[15] M. N. Nikiforova, R. A. Lynch, P. W. Biddinger et al., "RASPoint mutations and PAX8-PPAR $\gamma$ rearrangement in thyroid tumors: evidence for distinct molecular pathways in thyroid follicular carcinoma," The Journal of Clinical Endocrinology and Metabolism, vol. 88, no. 5, pp. 2318-2326, 2003.

[16] Y. Nachalon, S. Stern-Shavit, G. Bachar, J. Shvero, D. Limon, and A. Popovtzer, "Aggressive palliation and survival in anaplastic thyroid carcinoma," JAMA Otolaryngology-Head and Neck Surgery, vol. 141, no. 12, pp. 1128-1132, 2015.

[17] A. Mohebati, M. Dilorenzo, F. Palmer et al., "Anaplastic thyroid carcinoma: a 25-year single-institution experience," Annals of Surgical Oncology, vol. 21, no. 5, pp. 1665-1670, 2014. 
[18] J. W. Park, S. H. Choi, H. I. Yoon et al., "Treatment outcomes of radiotherapy for anaplastic thyroid cancer," Radiation Oncology Journal, vol. 36, no. 2, pp. 103-113, 2018.

[19] F. Bozorg-Ghalati and M. Hedayati, "Molecular biomarkers of anaplastic thyroid carcinoma," Current Molecular Medicine, vol. 17, no. 3, pp. 181-188, 2017.

[20] M. Rossing, "Classification of follicular cell-derived thyroid cancer by global RNA profiling," Journal of Molecular Endocrinology, vol. 50, no. 2, pp. R39-R51, 2013.

[21] M. N. Nikiforova, S. I. Chiosea, and Y. E. Nikiforov, "MicroRNA expression profiles in thyroid tumors," Endocrine Pathology, vol. 20, no. 2, pp. 85-91, 2009.

[22] C. S. Fuziwara and E. T. Kimura, "MicroRNAs in thyroid development, function and tumorigenesis," Molecular and Cellular Endocrinology, vol. 456, pp. 44-50, 2017.

[23] P. Pallante, R. Visone, M. Ferracin et al., "MicroRNA deregulation in human thyroid papillary carcinomas," Endocrine-related Cancer, vol. 13, no. 2, pp. 497-508, 2006.

[24] M. Swierniak, A. Wojcicka, M. Czetwertynska et al., "Indepth characterization of the microRNA transcriptome in normal thyroid and papillary thyroid carcinoma," The Journal of Clinical Endocrinology \& Metabolism, vol. 98, no. 8, pp. E1401-E1409, 2013.

[25] B. Wojtas, C. Ferraz, T. Stokowy et al., "Differential miRNA expression defines migration and reduced apoptosis in follicular thyroid carcinomas," Molecular and Cellular Endocrinology, vol. 388, no. 1-2, pp. 1-9, 2014.

[26] S.-Y. Sheu, F. Grabellus, S. Schwertheim, K. Worm, M. Broecker-Preuss, and K. W. Schmid, "Differential miRNA expression profiles in variants of papillary thyroid carcinoma and encapsulated follicular thyroid tumours," British Journal of Cancer, vol. 102, no. 2, pp. 376-382, 2010.

[27] T. Stokowy, B. Wojtaś, K. Fujarewicz, B. Jarząb, M. Eszlinger, and R. Paschke, "miRNAs with the potential to distinguish follicular thyroid carcinomas from benign follicular thyroid tumors: results of a meta-analysis," Hormone and Metabolic Research, vol. 46, no. 3, pp. 171-180, 2014.

[28] H. He, K. Jazdzewski, W. Li et al., "The role of microRNA genes in papillary thyroid carcinoma," Proceedings of the National Academy of Sciences, vol. 102, no. 52, pp. 1907519080, 2005.

[29] T. Stokowy, B. Wojtas, B. Jarzab et al., “Two-miRNA classifiers differentiate mutation-negative follicular thyroid carcinomas and follicular thyroid adenomas in fine needle aspirations with high specificity," Endocrine, vol. 54, no. 2, pp. 440-447, 2016.

[30] M. S. Dettmer, A. Perren, H. Moch, P. Komminoth, Y. E. Nikiforov, and M. N. Nikiforova, "MicroRNA profile of poorly differentiated thyroid carcinomas: new diagnostic and prognostic insights," Journal of Molecular Endocrinology, vol. 52, no. 2, pp. 181-189, 2014.

[31] S. M. Hammond, "An overview of microRNAs," Advanced Drug Delivery Reviews, vol. 87, pp. 3-14, 2015.

[32] M. T. Tetzlaff, A. Liu, X. Xu et al., "Differential expression of miRNAs in papillary thyroid carcinoma compared to multinodular goiter using formalin fixed paraffin embedded tissues," Endocrine Pathology, vol. 18, no. 3, pp. 163-173, 2007.

[33] T. Stokowy, M. Eszlinger, M. Świerniak et al., “Analysis options for high-throughput sequencing in miRNA expression profiling," BMC Research Notes, vol. 7, no. 1, p. 144, 2014.
[34] M. A. Newman, V. Mani, and S. M. Hammond, "Deep sequencing of microRNA precursors reveals extensive $3^{\prime}$ end modification," RNA, vol. 17, no. 10, pp. 1795-1803, 2011.

[35] J. Baran-Gale, E. E. Fannin, C. L. Kurtz, and P. Sethupathy, "Beta cell 5 '-shifted isomiRs are candidate regulatory hubs in type 2 diabetes," PLoS One, vol. 8, no. 9, Article ID e73240, 2013.

[36] C. Fernández-Hernando, C. M. Ramírez, L. Goedeke, and Y. Suárez, "MicroRNAs in metabolic disease," Arteriosclerosis, Thrombosis, and Vascular Biology, vol. 33, no. 2, pp. 178-185, 2013.

[37] M. D. Sacar Demirci, M. Yousef, and J. Allmer, "Computational prediction of functional MicroRNA-mRNA interactions," Computational Biology of Non-Coding RNA, Methods In Molecular Biology, Humana Press, vol. 1912, pp. 175-196, Humana Press, 2019.

[38] P. J. Mishra, "MicroRNAs as promising biomarkers in cancer diagnostics," Biomarker Research, vol. 2, no. 1, p. 19, 2014.

[39] P. J. Mishra, "Non-coding RNAs as clinical biomarkers for cancer diagnosis and prognosis," Expert Review of Molecular Diagnostics, vol. 14, no. 8, pp. 917-919, 2014.

[40] M. Acunzo, G. Romano, D. Wernicke, and C. M. Croce, "MicroRNA and cancer - a brief overview," Advances in Biological Regulation, vol. 57, pp. 1-9, 2015.

[41] G. Di Leva, M. Garofalo, and C. M. Croce, "MicroRNAs in cancer," Annual Review of Pathology: Mechanisms of Disease, vol. 9, no. 1, pp. 287-314, 2014.

[42] B. Liang, Y. Li, and T. Wang, "A three miRNAs signature predicts survival in cervical cancer using bioinformatics analysis," Scientific Reports, vol. 7, no. 1, p. 5624, 2017.

[43] G. A. Calin, C. D. Dumitru, M. Shimizu et al., "Nonlinear partial differential equations and applications: frequent deletions and down-regulation of micro- RNA genes miR 15 and miR16 at 13q14 in chronic lymphocytic leukemia," Proceedings of the National Academy of Sciences, vol. 99, no. 24, pp. 15524-15529, 2002.

[44] B. Gowrishankar, I. Ibragimova, Y. Zhou et al., "MicroRNA expression signatures of stage, grade, and progression in clear cell RCC," Cancer Biology \& Therapy, vol. 15, no. 3, pp. 329-341, 2014.

[45] H. Lee, K. Kim, N. Cho et al., "MicroRNA expression profiling and Notch1 and Notch2 expression in minimal deviation adenocarcinoma of uterine cervix," World Journal of Surgical Oncology, vol. 12, no. 1, p. 334, 2014.

[46] W. Li, X. Jin, Q. Zhang, G. Zhang, X. Deng, and L. Ma, "Decreased expression of miR-204 is associated with poor prognosis in patients with breast cancer," International Journal of Clinical and Experimental Pathology, vol. 7, no. 6, pp. 3287-3292, 2014.

[47] W. Bao, H.-H. Wang, F.-J. Tian et al., “A TrkB-STAT3-miR204-5p regulatory circuitry controls proliferation and invasion of endometrial carcinoma cells," Molecular Cancer, vol. 12, no. 1, p. 155, 2013

[48] Y. Yin, B. Zhang, W. Wang et al., "miR-204-5p inhibits proliferation and invasion and enhances chemotherapeutic sensitivity of colorectal cancer cells by downregulating RAB22A," Clinical Cancer Research, vol. 20, no. 23, pp. 6187-6199, 2014.

[49] M. N. Nikiforova, G. C. Tseng, D. Steward, D. Diorio, and Y. E. Nikiforov, "MicroRNA expression profiling of thyroid tumors: biological significance and diagnostic utility," The Journal of Clinical Endocrinology \& Metabolism, vol. 93, no. 5, pp. 1600-1608, 2008. 
[50] F. Marini, E. Luzi, and M. L. Brandi, "MicroRNA role in thyroid cancer development," Journal of Thyroid Research, vol. 2011, Article ID 407123, 12 pages, 2011.

[51] M. Celano, F. Rosignolo, V. Maggisano et al., "MicroRNAs as biomarkers in thyroid carcinoma," International Journal of Genomics, vol. 2017, Article ID 6496570, 11 pages, 2017.

[52] Z. Yang, Z. Yuan, Y. Fan, X. Deng, and Q. Zheng, "Integrated analyses of microRNA and mRNA expression profiles in aggressive papillary thyroid carcinoma," Molecular Medicine Reports, vol. 8, no. 5, pp. 1353-1358, 2013.

[53] J. C. Lee, J. T. Zhao, R. J. Clifton-Bligh et al., "MicroRNA-222 and microRNA-146b are tissue and circulating biomarkers of recurrent papillary thyroid cancer," Cancer, vol. 119, no. 24, pp. 4358-4365, 2013.

[54] J. C. Lee, J. S. Gundara, A. Glover, J. Serpell, and S. B. Sidhu, "MicroRNA expression profiles in the management of papillary thyroid cancer," The Oncologist, vol. 19, no. 11, pp. 1141-1147, 2014.

[55] Y. S. Lee, Y. S. Lim, J.-C. Lee et al., "Differential expression levels of plasma-derived miR-146b and miR-155 in papillary thyroid cancer," Oral Oncology, vol. 51, no. 1, pp. 77-83, 2015.

[56] F. Acibucu, H. Dökmetaş, Y. Tutar, Ş. Elagoz, and F. Kilicli, "Correlations between the expression levels of microRNA146b, 221, 222 and p27Kip1 protein mRNA and the clinicopathologic parameters in papillary thyroid cancers," Experimental and Clinical Endocrinology \& Diabetes, vol. 122, no. 3, pp. 137-143, 2014.

[57] L. Yip, L. Kelly, Y. Shuai et al., "MicroRNA signature distinguishes the degree of aggressiveness of papillary thyroid carcinoma," Annals of Surgical Oncology, vol. 18, no. 7, pp. 2035-2041, 2011.

[58] Y. Sun, S. Yu, Y. Liu, F. Wang, Y. Liu, and H. Xiao, "Expression of miRNAs in papillary thyroid carcinomas is associated with BRAF Mutation and clinicopathological features in Chinese patients," International Journal of Endocrinology, vol. 2013, Article ID 128735, 10 pages, 2013.

[59] E. R. Dorris, P. Smyth, J. J. O'Leary, and O. Sheils, “MIR141 expression differentiates hashimoto thyroiditis from PTC and benign thyrocytes in Irish archival thyroid tissues," Frontiers in Endocrinology, vol. 3, p. 102, 2012.

[60] M. Saiselet, D. Gacquer, A. Spinette et al., "New global analysis of the microRNA transcriptome of primary tumors and lymph node metastases of papillary thyroid cancer," BMC Genomics, vol. 16, no. 1, p. 828, 2015.

[61] M. Saiselet, J. M. Pita, A. Augenlicht et al., "miRNA expression and function in thyroid carcinomas: a comparative and critical analysis and a model for other cancers," Oncotarget, vol. 7, no. 32, pp. 52475-52492, 2016.

[62] T. Stokowy, D. Gawel, and B. Wojtas, "Differences in miRNA and mRNA profile of papillary thyroid cancer variants," International Journal of Endocrinology, vol. 2016, Article ID 1427042, 10 pages, 2016.

[63] Z.-L. Qiu, C.-T. Shen, H.-J. Song, W.-J. Wei, and Q.-Y. Luo, "Differential expression profiling of circulation microRNAs in PTC patients with non- ${ }^{131} \mathrm{I}$ and ${ }^{131} \mathrm{I}$-avid lungs metastases: a pilot study," Nuclear Medicine and Biology, vol. 42, no. 5, pp. 499-504, 2015.

[64] M. E. R. Graham, R. D. Hart, S. Douglas et al., "Serum microRNA profiling to distinguish papillary thyroid cancer from benign thyroid masses," Journal of OtolaryngologyHead \& Neck Surgery, vol. 44, no. 1, p. 33, 2015.
[65] A. A. Czajka, A. Wójcicka, A. Kubiak et al., "Family of microRNA-146 regulates RAR $\beta$ in papillary thyroid carcinoma," PLoS One, vol. 11, no. 3, Article ID e0151968, 2016.

[66] Y.-T. Chen, N. Kitabayashi, X. K. Zhou, T. J. Fahey 3rd., and T. Scognamiglio, "MicroRNA analysis as a potential diagnostic tool for papillary thyroid carcinoma," Modern $\mathrm{Pa}$ thology, vol. 21, no. 9, pp. 1139-1146, 2008.

[67] C.-K. Chou, R.-F. Chen, F.-F. Chou et al., "miR-146b is highly expressed in adult papillary thyroid carcinomas with high risk features including extrathyroidal invasion and the BRAFV $^{600 E}$ mutation," Thyroid, vol. 20, no. 5, pp. 489-494, 2010.

[68] C.-K. Chou, K. D. Yang, F.-F. Chou et al., "Prognostic implications of miR-146b expression and its functional role in papillary thyroid carcinoma," The Journal of Clinical Endocrinology \& Metabolism, vol. 98, no. 2, pp. E196-E205, 2013.

[69] X. Deng, B. Wu, K. Xiao et al., "MiR-146b-5p promotes metastasis and induces epithelial-mesenchymal transition in thyroid cancer by targeting ZNRF3," Cellular Physiology and Biochemistry, vol. 35, no. 1, pp. 71-82, 2015.

[70] M. V. Geraldo, A. S. Yamashita, and E. T. Kimura, "MicroRNA miR-146b-5p regulates signal transduction of TGF- $\beta$ by repressing SMAD4 in thyroid cancer," Oncogene, vol. 31, no. 15, pp. 1910-1922, 2012.

[71] N.-S. Ab Mutalib, S. N. Othman, A. Mohamad Yusof, S. N. Abdullah Suhaimi, R. Muhammad, and R. Jamal, "Integrated microRNA, gene expression and transcription factors signature in papillary thyroid cancer with lymph node metastasis," PeerJ, vol. 4, Article ID e2119, 2016.

[72] L. Liu, J. Wang, X. Li et al., "MiR-204-5p suppresses cell proliferation by inhibiting IGFBP5 in papillary thyroid carcinoma," Biochemical and Biophysical Research Communications, vol. 457, no. 4, pp. 621-626, 2015.

[73] L. Dai, Y. Wang, L. Chen, J. Zheng, J. Li, and X. Wu, "MiR221, a potential prognostic biomarker for recurrence in papillary thyroid cancer," World Journal of Surgical Oncology, vol. 15, no. 1, p. 11, 2017.

[74] S. Cantara, T. Pilli, G. Sebastiani et al., "Circulating miRNA95 and miRNA190 are sensitive markers for the differential diagnosis of thyroid nodules in a Caucasian population," The Journal of Clinical Endocrinology and Metabolism, vol. 99, no. 11, pp. 4190-4198, 2014.

[75] M. V. Geraldo, H. I. Nakaya, and E. T. Kimura, "Downregulation of $14 \mathrm{q} 32$-encoded miRNAs and tumor suppressor role for miR-654-3p in papillary thyroid cancer," Oncotarget, vol. 8, no. 6, pp. 9597-9607, 2017.

[76] A. Formosa, E. K. Markert, A. M. Lena et al., "MicroRNAs, miR-154, miR-299-5p, miR-376a, miR-376c, miR-377, miR381, miR-487b, miR-485-3p, miR-495 and miR-654-3p, mapped to the $14 \mathrm{q} 32.31$ locus, regulate proliferation, apoptosis, migration and invasion in metastatic prostate cancer cells," Oncogene, vol. 33, no. 44, pp. 5173-5182, 2014.

[77] A. Y. Mohamad, R. Jamal, R. Muhammad et al., "Integrated characterization of MicroRNA and mRNA transcriptome in papillary thyroid carcinoma," Frontiers in Endocrinology, vol. 9, p. 158, 2018.

[78] V. Mancikova, E. Castelblanco, E. Pineiro-Yanez et al., "MicroRNA deep-sequencing reveals master regulators of follicular and papillary thyroid tumors," Modern Pathology, vol. 28, no. 6, pp. 748-757, 2015.

[79] P. Agretti, E. Ferrarini, T. Rago et al., "MicroRNA expression profile helps to distinguish benign nodules from papillary thyroid carcinomas starting from cells of fine-needle 
aspiration,” European Journal of Endocrinology, vol. 167, no. 3, pp. 393-400, 2012.

[80] Z. Guo, H. Hardin, C. Montemayor-Garcia et al., "In situ hybridization analysis of miR-146b-5p and miR-21 in thyroid nodules: diagnostic implications," Endocrine Pathology, vol. 26, no. 2, pp. 157-163, 2015.

[81] M. Li, Q. Song, H. Li, Y. Lou, and L. Wang, "Circulating miR25-3p and miR-451a may be potential biomarkers for the diagnosis of papillary thyroid carcinoma," PLoS One, vol. 10, no. 7, Article ID e0132403, 2015.

[82] A. Sondermann, F. M. Andreghetto, A. C. B. Moulatlet et al., "MiR-9 and miR-21 as prognostic biomarkers for recurrence in papillary thyroid cancer," Clinical \& Experimental Metastasis, vol. 32, no. 6, pp. 521-530, 2015.

[83] T. Liu, X. You, J. Sui et al., "Prognostic value of a twomicroRNA signature for papillary thyroid cancer and a bioinformatic analysis of their possible functions," Journal of Cellular Biochemistry, vol. 120, no. 5, pp. 7185-7198, 2018.

[84] Y.-L. Zhou, C. Liu, X.-x. Dai, X.-H. Zhang, and O.-C. Wang, "Overexpression of miR-221 is associated with aggressive clinicopathologic characteristics and the BRAF mutation in papillary thyroid carcinomas," Medical Oncology, vol. 29, no. 5, pp. 3360-3366, 2012.

[85] M. V. Geraldo, C. S. Fuziwara, C. U. M. Friguglieti et al., "MicroRNAs miR-146-5p and let-7f as prognostic tools for aggressive papillary thyroid carcinoma: a case report," Arquivos Brasileiros de Endocrinologia and Metabologia, vol. 56, no. 8, pp. 552-557, 2012.

[86] R. Samsonov, V. Burdakov, T. Shtam et al., "Plasma exosomal miR-21 and miR-181a differentiates follicular from papillary thyroid cancer," Tumor Biology, vol. 37, no. 9, pp. 12011-12021, 2016.

[87] F. Weber, R. E. Teresi, C. E. Broelsch, A. Frilling, and C. Eng, "A limited set of human MicroRNA is deregulated in follicular thyroid carcinoma," The Journal of Clinical Endocrinology \& Metabolism, vol. 91, no. 9, pp. 3584-3591, 2006.

[88] T. Stokowy, B. Wojtaś, J. Krajewska et al., "A two miRNA classifier differentiates follicular thyroid carcinomas from follicular thyroid adenomas," Molecular and Cellular Endocrinology, vol. 399, pp. 43-49, 2015.

[89] M. Colamaio, G. Calì, D. Sarnataro et al., "Let-7a downregulation plays a role in thyroid neoplasias of follicular histotype affecting cell adhesion and migration through its ability to target the FXYD5 (dysadherin) gene," The Journal of Clinical Endocrinology \& Metabolism, vol. 97, no. 11, pp. E2168-E2178, 2012.

[90] M. Colamaio, F. Puca, E. Ragozzino et al., "miR-142-3p down-regulation contributes to thyroid follicular tumorigenesis by targeting ASH1L and MLL1," The Journal of Clinical Endocrinology \& Metabolism, vol. 100, no. 1, pp. E59-E69, 2015.

[91] R. Visone, P. Pallante, A. Vecchione et al., "Erratum: specific microRNAs are downregulated in human thyroid anaplastic carcinomas," Oncogene, vol. 35, no. 39, p. 5214, 2016.

[92] S. T. Aherne, P. Smyth, M. Freeley et al., "Altered expression of mir-222 and mir-25 influences diverse gene expression changes in transformed normal and anaplastic thyroid cells, and impacts on MEK and TRAIL protein expression," International Journal of Molecular Medicine, vol. 38, no. 2, pp. 433-445, 2016.

[93] M. Boufraqech, N. Nilubol, L. Zhang et al., "miR30a inhibits LOX expression and anaplastic thyroid cancer progression," Cancer Research, vol. 75, no. 2, pp. 367-377, 2015.
[94] J. Braun, C. Hoang-Vu, H. Dralle, and S. Hüttelmaier, "Downregulation of microRNAs directs the EMT and invasive potential of anaplastic thyroid carcinomas," Oncogene, vol. 29, no. 29, pp. 4237-4244, 2010.

[95] P. A. Gregory, A. G. Bert, E. L. Paterson et al., "The miR-200 family and miR-205 regulate epithelial to mesenchymal transition by targeting ZEB1 and SIP1," Nature Cell Biology, vol. 10, no. 5, pp. 593-601, 2008.

[96] S.-M. Park, A. B. Gaur, E. Lengyel, and M. E. Peter, "The miR-200 family determines the epithelial phenotype of cancer cells by targeting the E-cadherin repressors ZEB1 and ZEB2," Genes \& Development, vol. 22, no. 7, pp. 894-907, 2008.

[97] U. Burk, J. Schubert, U. Wellner et al., "A reciprocal repression between ZEB1 and members of the miR-200 family promotes EMT and invasion in cancer cells," EMBO Reports, vol. 9, no. 6, pp. 582-589, 2008.

[98] Q. Bu, F. You, G. Pan et al., "MiR-125b inhibits anaplastic thyroid cancer cell migration and invasion by targeting PIK3CD," Biomedicine \& Pharmacotherapy, vol. 88, pp. 443-448, 2017.

[99] J. H. E. Mäki-Jouppila, S. Pruikkonen, M. B. Tambe et al., "MicroRNA let-7b regulates genomic balance by targeting Aurora B kinase," Molecular Oncology, vol. 9, no. 6, pp. 1056-1070, 2015.

[100] S. Schwertheim, S.-Y. Sheu, K. Worm, F. Grabellus, and K. W. Schmid, "Analysis of deregulated miRNAs is helpful to distinguish poorly differentiated thyroid carcinoma from papillary thyroid carcinoma," Hormone and Metabolic Research, vol. 41, no. 6, pp. 475-481, 2009.

[101] A. Fassina, R. Cappellesso, F. Simonato et al., "A 4-MicroRNA signature can discriminate primary lymphomas from anaplastic carcinomas in thyroid cytology smears," Cancer Cytopathology, vol. 122, no. 4, pp. 274-281, 2014.

[102] S. Takakura, N. Mitsutake, M. Nakashima et al., "Oncogenic role of miR-17-92 cluster in anaplastic thyroid cancer cells," Cancer Science, vol. 99, no. 6, pp. 1147-1154, 2008.

[103] Y. Xiong, L. Zhang, and E. Kebebew, "MiR-20a is upregulated in anaplastic thyroid cancer and targets LIMK1," PLoS One, vol. 9, no. 5, Article ID e96103, 2014.

[104] C. S. Fuziwara and E. T. Kimura, "MicroRNA deregulation in anaplastic thyroid cancer biology," International Journal of Endocrinology, vol. 2014, Article ID 743450, 8 pages, 2014.

[105] V. Haghpanah, P. Fallah, R. Tavakoli et al., "Antisense-miR21 enhances differentiation/apoptosis and reduces cancer stemness state on anaplastic thyroid cancer," Tumor Biology, vol. 37, no. 1, pp. 1299-1308, 2016.

[106] D. Abraham, N. Jackson, J. S. Gundara et al., "MicroRNA profiling of sporadic and hereditary medullary thyroid cancer identifies predictors of nodal metastasis, prognosis, and potential therapeutic targets," Clinical Cancer Research, vol. 17, no. 14, pp. 4772-4781, 2011.

[107] C. Mian, G. Pennelli, M. Fassan et al., "MicroRNA profiles in familial and sporadic medullary thyroid carcinoma: preliminary relationships with RET status and outcome," Thyroid, vol. 22, no. 9, pp. 890-896, 2012.

[108] G. Pennelli, F. Galuppini, S. Barollo et al., "The PDCD4/miR21 pathway in medullary thyroid carcinoma," Human $\mathrm{Pa}$ thology, vol. 46, no. 1, pp. 50-57, 2015.

[109] L. Santarpia, G. A. Calin, L. Adam et al., "A miRNA signature associated with human metastatic medullary thyroid carcinoma," Endocrine-related Cancer, vol. 20, no. 6, pp. 809-823, 2013. 
[110] J. Hudson, E. Duncavage, A. Tamburrino et al., "Overexpression of miR-10a and miR-375 and downregulation of YAP1 in medullary thyroid carcinoma," Experimental and Molecular Pathology, vol. 95, no. 1, pp. 62-67, 2013.

[111] Y.-H. Chu, H. Hardin, D. F. Schneider, H. Chen, and R. V. Lloyd, "MicroRNA-21 and long non-coding RNA MALAT1 are overexpressed markers in medullary thyroid carcinoma," Experimental and Molecular Pathology, vol. 103, no. 2, pp. 229-236, 2017.

[112] P. Romeo, C. Colombo, R. Granata et al., "Circulating miR375 as a novel prognostic marker for metastatic medullary thyroid cancer patients," Endocrine-related Cancer, vol. 25, no. 3, pp. 217-231, 2018.

[113] A. F. Christopher, R. P. Kaur, G. Kaur, A. Kaur, V. Gupta, and P. Bansal, "MicroRNA therapeutics: discovering novel targets and developing specific therapy," Perspectives in Clinical Research, vol. 7, no. 2, pp. 68-74, 2016.

[114] Z. Li and T. M. Rana, "Therapeutic targeting of microRNAs: current status and future challenges," Nature Reviews Drug Discovery, vol. 13, no. 8, pp. 622-638, 2014.

[115] A. Wójcicka, M. Kolanowska, and K. Jażdżewski, "Mechanisms IN endocrinology: microRNA in diagnostics and therapy of thyroid cancer," European Journal of Endocrinology, vol. 174, no. 3, pp. R89-R98, 2016.

[116] E. Bonneau, B. Neveu, E. Kostantin, G. J. Tsongalis, and V. De Guire, "How close are miRNAs from clinical practice? a perspective on the diagnostic and therapeutic market," EJIFCC, vol. 30, no. 2, pp. 114-127, 2019.

[117] R. Suresh, S. Sethi, S. Ali, T. Giorgadze, and F. H. Sarkar, "Differential expression of MicroRNAs in papillary thyroid carcinoma and their role in racial disparity," Journal of Cancer Science \& Therapy, vol. 7, no. 5, pp. 145-154, 2015. 